\title{
Improved NEPA Substations On-Line Monitoring Technology Using Optical Fiber Sensors
}

\author{
M. A. Omidiora \\ Electrical and Electronic Engineering Department \\ Ladoke Akintola University of Technology, P.M.B. 4000 \\ Ogbomoso, Oyo State, Nigeria
}

Received:April 16, $2003 \quad$ Accepted: May 19, 2003

\begin{abstract}
Condition monitoring of power substations is a significant issue for the National Electric Power Authority (NEPA) of Nigeria. Current methods for monitoring the condition of high voltage equipment are time consuming and often inaccurate, therefore causing catastrophic damage to the substation equipment. Recent research has demonstrated the efficacy of employing optical fiber sensors in condition monitoring of substation equipment. These sensors enhance the current monitoring method and facilitate faster responses to equipment problems. This paper briefly reviews the state of the arts, but is primarily devoted to a discussion of the new approach and future directions in condition monitoring of NEPA substation equipment.
\end{abstract}

\section{INTRODUCTION}

Nigeria's National Electric Power Authority (NEPA) is constantly under economic pressure to reduce costs while improving services, productivity and the safety of power system. To do this, predictive maintenance (PM) practices are increasingly becoming an important asset management tool. As a consequence, infrastructure, equipment and machine operators are looking to on-line, continuous monitoring systems to replace fixed-interval scheduled maintenance practices. The condition monitoring $(\mathrm{CM})$ of materials, structures and machinery involves the continuous analysis of structural components and equipment. The identification of problems before component breakage or machinery failure, involves three major techniques [1]: vibration monitoring, by-product monitoring, and performance monitoring. PM practices help estimate the time-to-breakdown, and thus help equipment to be maintained as required and taken off-line only as necessary [2].

With the increasing application of $\mathrm{CM}$ and PM practices, it is extremely important that the machinery and equipment characteristics are well understood, that the operation of machinery be optimized to reduce costs while improving product and service quality, and that a reliable monitoring system be incorporated within the equipment. Optical fiber technology offers a means by which these functions can be performed through the use of optical fiber sensors [3].

Optical fiber sensors though a relatively new technology in Nigeria have been gaining much attention. Optical sensors are particularly well suited for conditioning and monitoring applications in that they are lightweight, durable, and can be incorporated within equipment without being intrusive. The potential uses of optical fiber sensors are monitoring such electrical parameters as current and voltage and other parameters such as mechanical vibration. Wear and degradation analysis would also be possible using optical fibers to determine viscosity, acidity, degradation by-products, and wear or by using the fibers to collect near-infrared spectra [3].

In this paper, the functions, responsibilities and the basic architecture of substations within the NEPA national grid 
system are discussed in next section. Section three discusses the basic principle of optical fiber transmission, section four looks into the effects of using an optically controlled sensing technology in developing countries in Africa, with emphasis on its performance evaluation, and including a noise and cost benefit analysis. Lastly, the design and implementation of this technology for the NEPA system are highlighted in section five, before the conclusion.

\section{NEPA AND ITS SUBSTATIONS ARCHITECTURE}

a. Functions and Responsibilities of NEPA in Nigeria

The National Electric Power Authority (NEPA) in Nigeria is responsible for maintaining an efficient, harmonized and economic system of electricity supply to all parts of the nation. NEPA has become the largest and fastest growing electricity industry in Africa and, indeed, is one of the largest in the developing world with a consumer population of more than five million. NEPA has made great progress in the production and marketing of electricity to Nigeria and several neighboring countries. The Republic of Niger, through NIGERLEC (Niger Electric Company) has been a beneficiary of NEPA's extended electricity program, as has also Communaute Electricque du Benin (CEB), which is responsible for production and transmission of electric power in the Republic of Benin and Togo.

\section{b. Architecture of a NEPA Substation}

A typical NEPA substation can be described as an assembly of electrical equipment and apparatus which transforms electrical alternating current $A C$ ) from one voltage to another. Substations are vital links in any power system and are crucial in delivering electrical power from one of the Nigerian generating stations (Kainji, Jebba, Sapele, or Egbin, etc) to consumers both within and outside the country [4].

NEPA substations can be divided into the following four categories, as shown in Table 1.

\begin{tabular}{|l|l|}
\hline $\begin{array}{c}\text { Substation } \\
\text { Category }\end{array}$ & \multicolumn{1}{c|}{$\begin{array}{c}\text { Voltage } \\
\text { Transformation }\end{array}$} \\
\hline Step-Up & 16 to 330 kilovolts (KV) \\
\hline Primary & 330 to $132 \mathrm{KV}$ \\
\hline Secondary & 132 to $33 \mathrm{KV}$ \\
\hline Distribution & $\begin{array}{l}\text { (i) } 33 \text { to } 11 \mathrm{KV} \\
\text { (ii) } 11 \mathrm{KV} \text { to } 415 \text { volts }\end{array}$ \\
\hline
\end{tabular}

Table 1. NEPA Substation Categories

Figure 1 shows each category of substation within a simplified block diagram of the NEPA power grid. Equipment typically found in NEPA substations includes the following: switch gears, high rupturing capacity fuses (HRC), air-cored ring shielded reactors, lightening arresters, circuit breakers, power transformers, isolators, and bus bars, among other items.

\section{BASIC PRINCIPLES OF OPTICAL FIBER TRANSMISSION}

Optical fibers transmit information by turning electrical signals into light. Here light refers to more than the portion of the electromagnetic spectrum that is visible to the human eye [5].

The principles behind optical fiber systems are relatively simple. As shown in Figure 2 (a), optical fiber links contain three basic elements; the transmitter that allows for data input and outputs an optical signal, the optical fiber that carries the data, and the receiver that decodes the optical signal to output data.

The transmitter, shown in Figure 2 (b), uses an electrical interface, either video, audio, data, or another form of electrical input, to encode the user's information through frequency or chromatic modulation [1]. The electrical output of the modulator is usually transformed into light either by means of a light emitting diode (LED) or a laser diode (LD). The wavelengths of these light sources range from 660 nanometers $(\mathrm{nm})$ to $1550 \mathrm{~nm}$ for most optical fiber applications [5].

The receiver, illustrated in Figure 2 (c), decodes the light signal back into electrical signals. Two types of light detector 
are typically used: the PIN photodiode or the avalanche photodiode (APD). The detected and amplified electrical signal is then sent through a data decoder or demodulator that converts the electrical signal back into video, audio, or another form of user output.

\section{EFFECTS OF USING OPTICAL FIBER TECHNOLGY}

One attraction of optical fiber sensors is their intrinsic robustness in hostile and challenging environment [6]. Their ruggedness and flexibility mean that they can act as conduits into previously inaccessible areas and, even further, that the effect of an external variable on the fiber can be sensed via a number of very precise changes in the light beams within the fiber [7]. For high voltage, power apparatus applications, these properties include: immunity from electromagnetic interference, no electrical shock hazard, small size and lightweight character, among others.

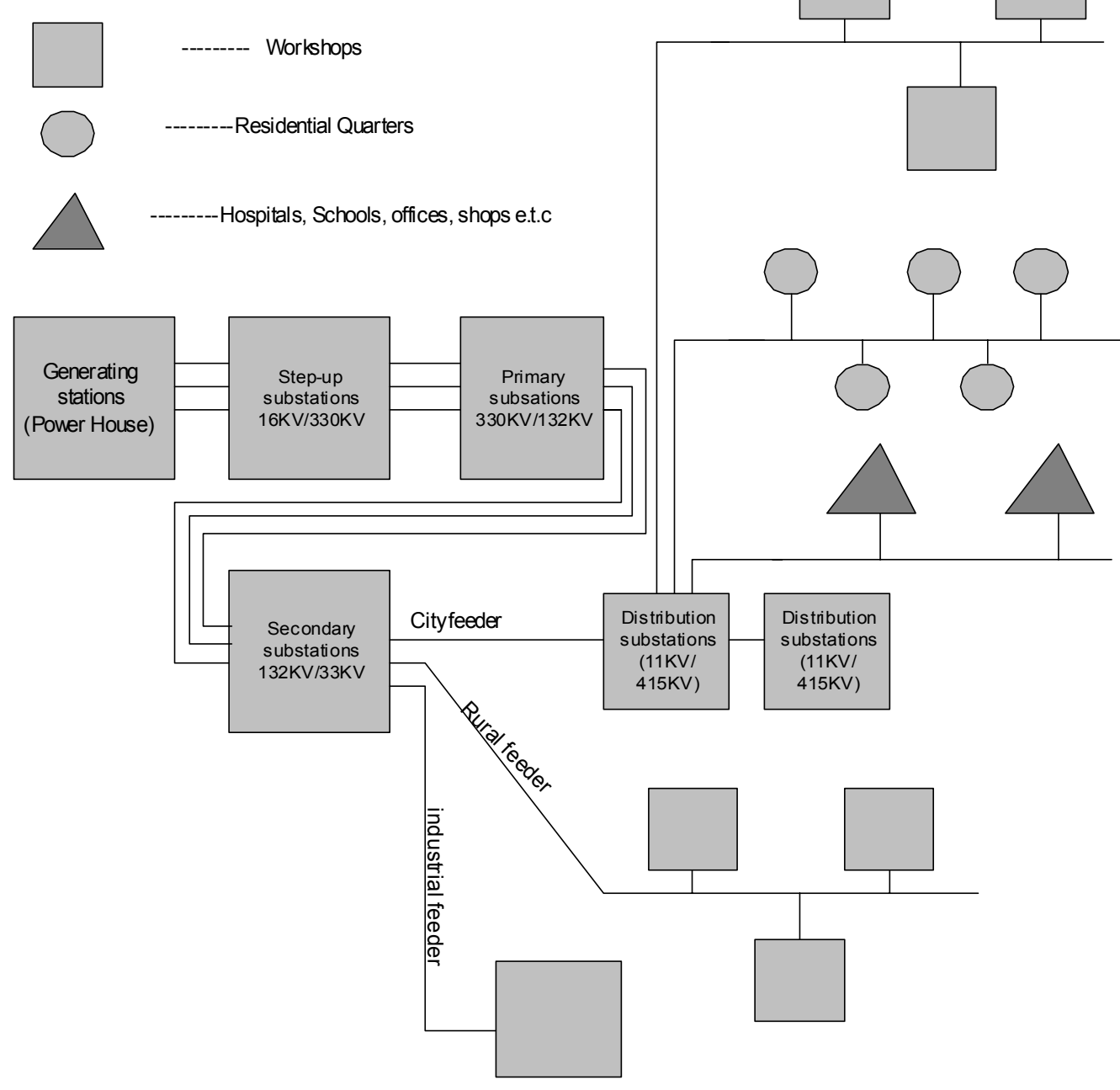

Figure 1. Simplified block diagram of substations in the NEPA transmission and distribution power system. 


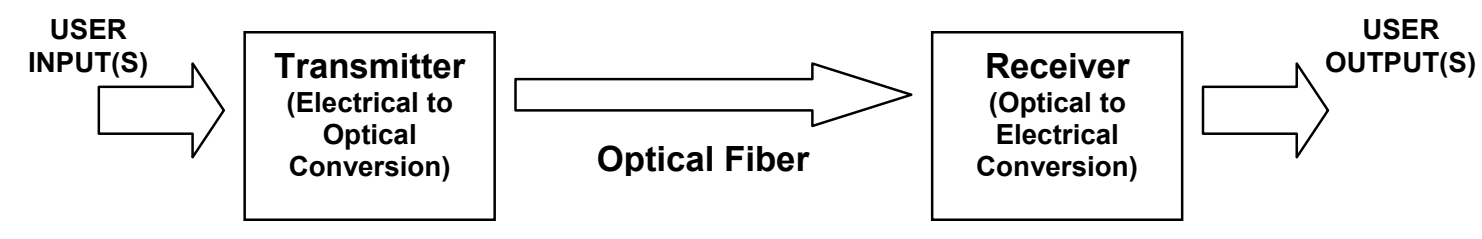

(a)

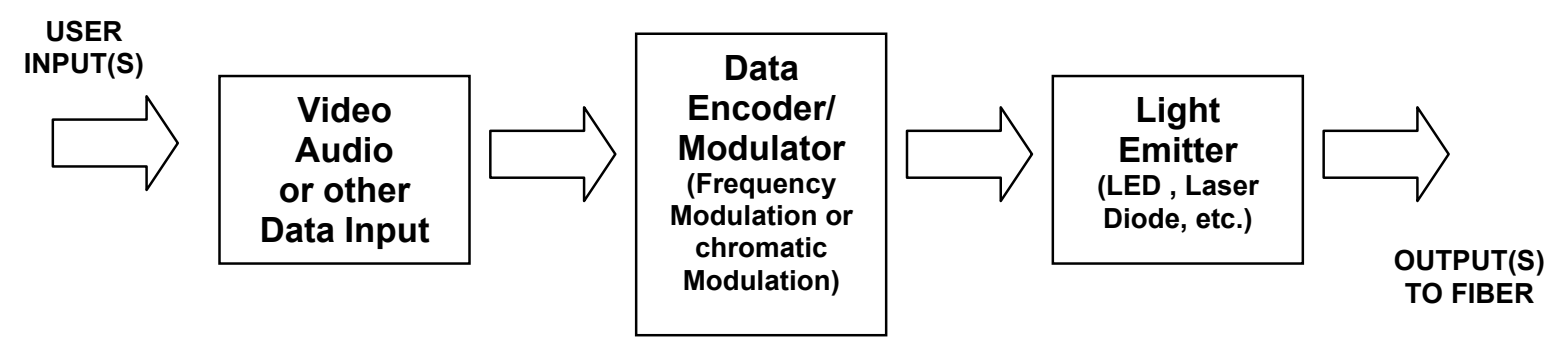

(b)

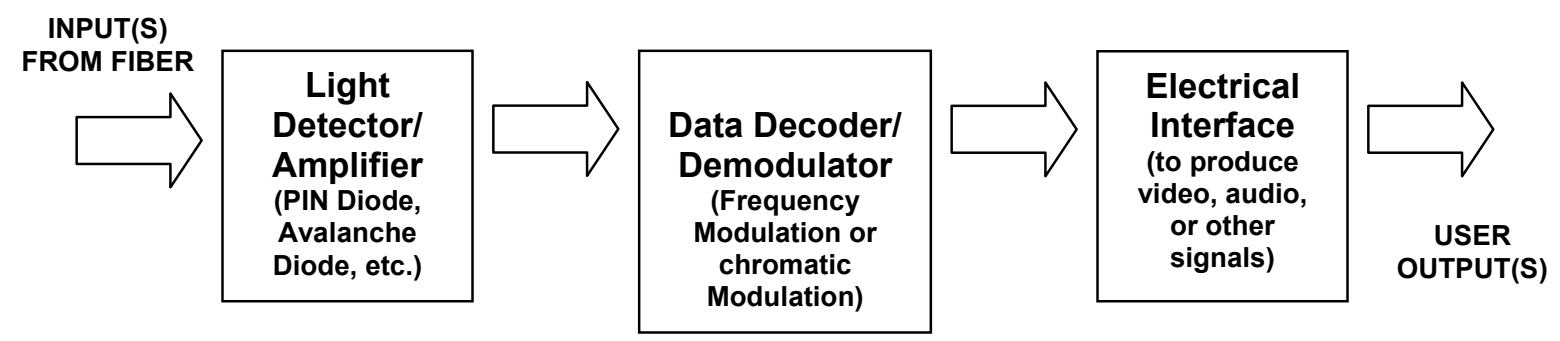

(c)

Figure 2. (a) Elements of Optical Fiber Transmission; (b) an Optical Fiber Transmitter; (c) an Optical Fiber Receiver [8]

Despite such attractive advantages, the innovative use of fiber sensing technology in developing countries in Africa, and especially Nigeria, has been retarded because of various constraints including end-user unfamiliarity with fiber technology, other more familiar technologies for monitoring systems, and an absence of established markets.

\section{a. Performance Evaluation of a Typical Optical Fiber Sensor}

A typical example of sensors that can be employed to monitor the condition of substations is an optical fiber chemical sensors system. The insulation system of a high voltage power or circuit transformer consists of cellulose paper and a processed mineral oil. Hydrolytic oxidation and 
environmental temperatures largely influence degradation of the insulating paper. The paper's condition can be tested using dissolved gas analysis (DGA) of the oil. A key indicator of the paper insulation breakdown in a power transformer is the formation of water, $\mathrm{CO}_{2}, \mathrm{CH}_{4}$ and several other known chemical compounds [4]. Although this insulation breakdown can be tested by DGA, the test must be carried out in a laboratory due to the large size, complexity and cost of the gas chromatography instrument used. Furthermore, it involves detailed procedures for sampling and testing. To overcome the disadvantages associated with DGA as currently used in most developing African nations' power industries and to effectively monitoring the operation of these highly expensive power transformers, optical fiber chemical sensors system can be employed for on-line monitoring, preferably from a central control.

\section{b. Cost/Benefit Analysis of the Technology}

The cost of optical fiber sensing system can be a critical consideration. While minimizing the cost is often a primary coal, there are performance trade-offs to consider. The components consideration such as light emitter type, emitter wavelength, connector type, fiber sensors type, detector type and others, will have an impact on both the cost and the performance of the system. Common sense goes a long way in designing the most costeffective system to meet an applications requirement. A practically engineered system is often the one that meets the required performance limits and margins with little extra to spare. Excess performance will often make the system too costly for the application.

\section{DESGIN AND IMPLEMENTATION OF AN OPTICAL FIBER SYSTEM}

One objective of the realization of optical fiber sensing in the NEPA system is the design and implementation of optically controlled substation systems. Such an objective involves producing optical fiber sensors for monitoring a range of parameters governing the condition and operation of power equipment such as circuit breakers and transformers. It entails interlinking the various monitoring systems with an optical fiber system and connecting these to various data stations and control units.

\section{a. Monitoring Electrical Parameters}

The emergence of new forms of current and voltage monitors are examples of fiber sensors that respect the real needs of the National Electric Power Authority in Nigeria and other electric power industries in Africa. Two main methods may be identified, corresponding to direct optical transduction of the current/voltage signal and to sensing via intermediate electronic transduction (hybrid transduction) [9].

Initially, the attraction was for purely optically monitoring relying on Faraday rotation and the pockets effect for current and voltage transduction, respectively [10, 11]. Such methods may be further subdivided into intrinsic systems, which utilize the transmitting fiber itself as the sensor, and extrinsic systems, which utilize separate, localized-sensing elements (see Figures 3 and 4, respectively).

One penalty associated with the intrinsic approach is poor optical processing. Attempts to stabilize the systems by compensation are cumbersome, complex and costly. The complexity and unreliability of the intrinsic approach may be alleviated to a large extent through the use of the extrinsic approach in conjunction with chromatic monitoring techniques. The benefits in system simplicity (and hence cost and reliability) that accrue are apparent from a comparison of Figures 3 and 4 . The need for special fibers and intermediate optical components (Wollaston prisms, fiber splitters etc.) is removed through the use of the chromatic extrinsic system [6]. Nonetheless, the optical sensing elements of such systems remain susceptible to temperature and vibrational instabilities.

The simplicity of the optical sensing system may be further reduced, and the difficulties associated with the stability of the Electro-optic elements overcome, through the use of a hybrid system. Hybrid systems (Figure 5) use current-voltage transformers for the intermediate transduction into a suitable 


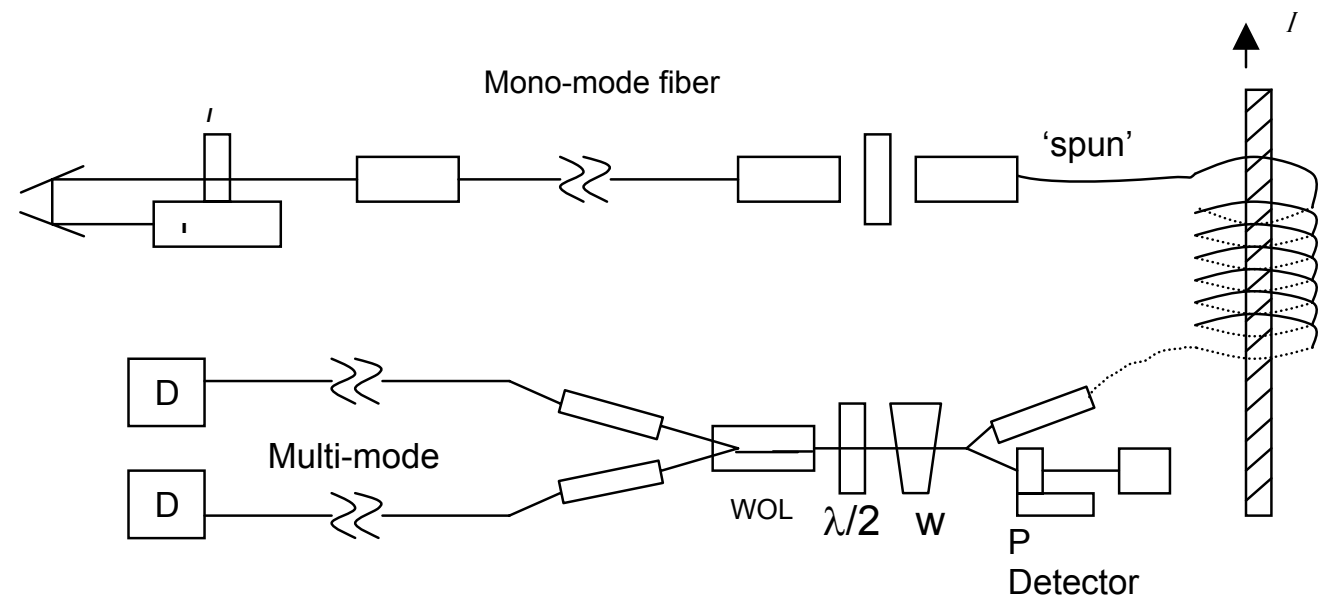

Figure 3. Intrinsic Electro-Optic Sensing system, where $L=$ Laser; $D=$ Detector; $P=$ Polarizer; $W$ = beam splitter; $\mathrm{WOL}=$ Wollaston Prism

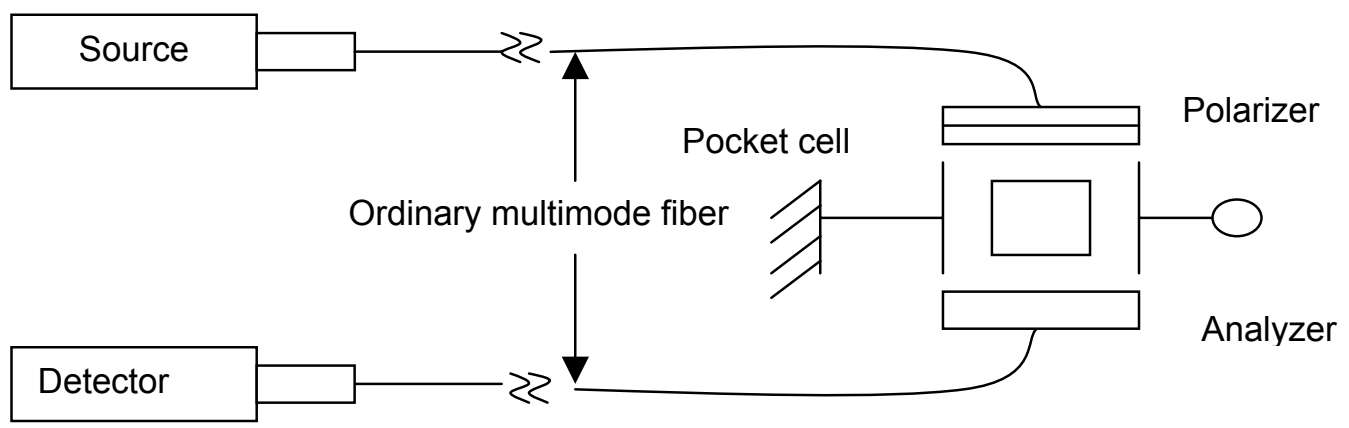

Figure 4. Extrinsic Electro-Optic Sensing system

electronic form for optical modulation [6]. The systems have the added advantage, therefore, of being able to be retrofitted on existing transformers already installed in power systems.

Because of the inherent weak coupling between the optical signals within the fibers and electrical devices, the currentvoltage transformer insulation requirement may be relaxed leading to further reduction in cost and size. The replacement of wire by fiber connections also avoids electromagnetic pick-up between signals from different power systems, providing weight reduction advantages and automatic electronic isolation [9]. Finally, the optically linked current transformer is more compatible in producing digital input for microprocessors, which are being increasingly used for controlling protective functions on power equipment.

b. Monitoring Non-Electrical Equipment

Notwithstanding the importance of monitoring electrical parameters such as voltage and current, there is also a major requirement for monitoring mechanical and thermal values on power equipment since failure often affects these parameters. For instance, a majority of power switchgear and circuit breaker faults are mechanical in nature [3]. Vibration and the associated frequency analysis have become very popular monitoring techniques due to the myriad of information that they can provide 


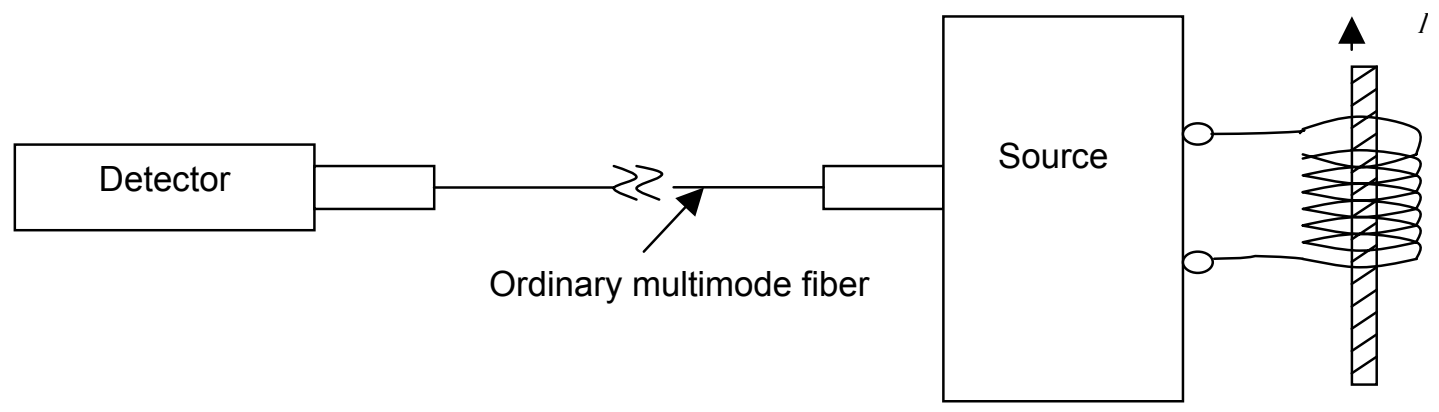

Figure 5. A Hybrid Electro-Optic Sensing system

about the condition of structures and mechanical misalignment. Vibrational and frequency analyses of machinery can be very useful for the detection of imbalance, mechanical looseness, bearing defects, van/blade pass problems, electric motor defect or uneven loading of a machine. Furthermore, vibrational analysis is a very effective method for monitoring undesirable high vibrational levels, which affect machine health and lead to structural fatigue problems [12].

Optical fiber based systems are available, and have already undergone site testing, for monitoring parameters such as pressure and temperature of gas-filled equipment (circuit breakers, and gas insulated bus-bar chambers), mechanical linkages on switch-gear and occurrences of arcing [13].

The mass density of gas in sulphur hexafluoride filled equipment may be monitored with a system produced by Kent Instruments (Figure 6) [2]. This is an example of a frequency-modulated system, whereby the natural frequency of vibration of a wire attached to a pressure-sensitive diaphragm is modulated by the gas density. The vibration of the wire is excited by pulsed optical power transmission via a fiber link and the vibrations are detected by the shuttering action of the wire on a fiber data link. This sensor system is very sensitive to pressure and temperature changes.

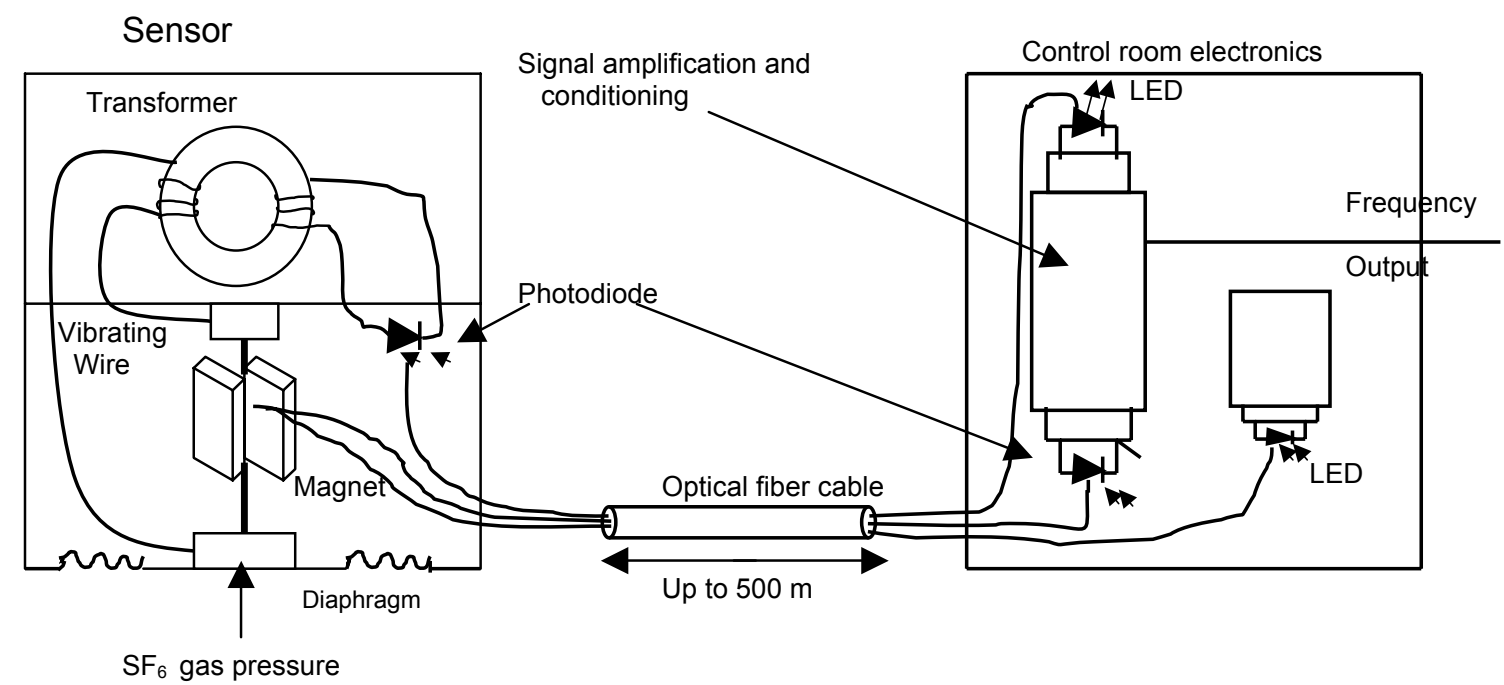

Figure 6. Schematic diagram of the Kent Instruments P901 Gas Density Sensor [3]. 


\section{CONCLUSION}

Optical fiber monitoring has evolved beyond scientific demonstrations to the engineering optimization and commercial viability phase [3]. Optical fiber sensors possess several advantages over many existing conventional sensors and are highly gaining attention in power industries [7]. The cost of optical fiber systems is rapidly decreasing and sensing techniques are being refined. As a result, this technology will offer a reliable and inexpensive measurement tool in many sensing applications in the Nigerian power industry if it can be implemented in the NEPA system.

The extensive use of optical fiber sensing technologies for the monitoring and diagnosis of the condition and performance of heavy power equipment in NEPA substations could provide a sound engineering and economic basis for the major decisions which will have to be made concerning the operation, maintenance, refurbishment or replacement and life extension of these large items. The savings made by avoiding - or delaying-forced outages and the resulting refurbishment or replacement could be substantial. The improvement in safety, performance and reliability of NEPA substation equipment using this new technology would also have great potential to the system and society as a whole.

\section{ACKNOWLEDGEMNT}

I gratefully acknowledge the academic assistance and advice of Mr. Dele Oluwade, Mr. Tunde Olabiyisi and Mr. Musa Awoyemi, and wish to express my gratitude for the support of my brother, Mr. Omidiora E. Olusayo during the preparation of this paper.

\section{REFERENCES}

1. P. Ball, Achieving Reliability - A Basic Understanding Process, Proceedings: (CM) 2 Forum, pp. 1-13, 1994.

2. L. Simmon, Introduction to Plant Condition Monitoring, VIPAC Occasional Paper No. 14, pp. 1-4, 1993.

3. E. E. Tapanes, I. Oanca, J. Katsifoils, J. Goode, And Q. Su, The Innovate Use of Optical Fibers for Condition Monitoring of High Voltage Equipment,
Proceedings: IEEE Optimization of Romania, pp. 791-814, May 1996.

4. O. Aduloju, Electrical Power Systems, Vol. 1 (Evidence Nigeria Ventures, llorin, Nigeria, 1999).

5. J. J. Collins, Fundaments of Optical Fibre Transmission (Chapman and Hall, London, 1983).

6. John M. Senior, Optical Fiber Communication, Principle and Practice (Prentice Hall, New York, 1985).

7. Charles K. Kao, Optical Fibre (Institution of Electrical Engineers (UK), Peter Peregrinus, London, 1988).

8. David R. Goff, Fiber Optic Reference Guide (Focal Press, Burlington, Massachusetts, USA, 1996).

9. Hiroshi Murata, Handbook of Optical Fibers and Cable (Marcel Dekker, New York, 1988).

10. Paul N. Butcher and David Cottar, The Elements of Nonlinear Optics (Cambridge University Press, New York, 1991).

11. P. Yeh and C. Gu, "Optical Activity and Faraday Rotation." §3.6 in Optics of Liquid Crystal Displays. (Wiley, New York, New York, USA, 1999) pp. 75-86. Also: http://scienceworld.wolfram.com/ physics/FaradayRotation.html

12. Alan Foster, Electrical Supply, No. 13, pp. 4-7, December 1993.

13. N. F. Rieger, T. H. McClosky, and R. P. Dewey, The High Cost of Failure of Rotation Equipment, Proceedings (CM) 2 Forum, pp. 171-182, 1994.

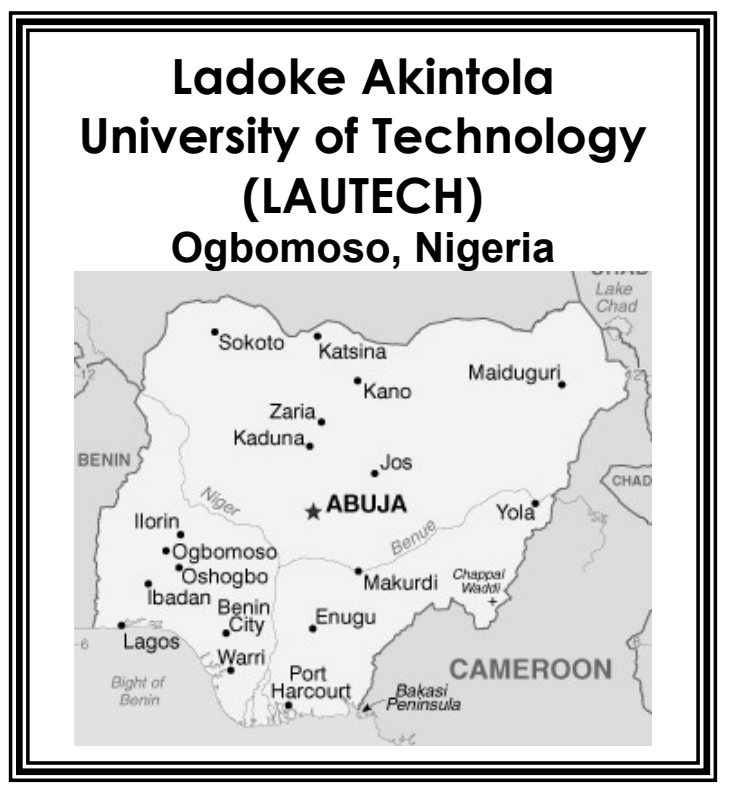

